REGULADORES DE LA BROTACIÓN, ALTERNATIVOS A LA CIANAMIDA HIDROGENADA, PARA SUPLIR LA FALTA DE HORAS DE FRÍO EN VIÑEDOS TROPICALES DEL LITORAL ECUATORIANO.

Raquel González-Herranz, Lourdes Ortega 


\title{
REGULADORES DE LA BROTACIÓN, ALTERNATIVOS A LA CIANAMIDA HIDROGENADA, PARA SUPLIR LA FALTA DE HORAS DE FRÍO EN VIÑEDOS TROPICALES DEL LITORAL ECUATORIANO.
}

\author{
González-Herranz, Raquel ${ }^{1}$; Ortega, Lourdes ${ }^{1}$ \\ ${ }^{1}$ CIAP-UPSE. Universidad Estatal Península de Santa Elena (UPSE) \\ Campus La Libertad, vía principal Santa Elena - La Libertad. La Libertad-Ecuador. \\ E-mail: raquel.gonzalez.herranz@gmail.com
}

\begin{abstract}
Resumen
La cianamida hidrogenada $(\mathrm{CH})$ es un eficaz estimulante de la brotación de las yemas habitualmente utilizado en viticultura tropical, pero manipular esta materia activa conlleva un alto riesgo por su elevada toxicidad. Según algunos estudios tratamientos alternativos podrían llegar a producir sobre las yemas efectos similar a la $\mathrm{CH}$, compensado la falta de horas de frío en viñedos situados en zonas tropicales, como los ubicados en la costa sur ecuatoriana. De entre todos los tratamientos evaluados destacan los efectos producidos por la aplicación directa sobre las yemas de peróxido de hidrógeno al 5\%. Este tratamiento consigue estimular la brotación de la práctica totalidad de las yemas, adelantando e incrementando el desarrollo vegetativo y duplicando el rendimiento de las cepas, sin mermar la calidad de los racimos producidos. Por tanto podemos afirmar que los tratamientos con peróxido de hidrógeno son una alternativa viable, más económica y sostenible, a las aplicaciones de $\mathrm{CH}$ en estas condiciones de cultivo
\end{abstract}

Palabras Claves: fitorreguladores, peróxido de hidrogeno, yema, toxicidad, producción

\begin{abstract}
Hydrogen cyanamide $(\mathrm{CH})$ is an effective stimulant bud breaking usually used in tropical viticulture, but handling this active substance carries a high risk because of their high toxicity. According to some studies alternative treatments could potentially produce a similar effect on the buds to $\mathrm{CH}$, offset the lack of chilling hours in vineyards located in areas without a sufficient period of chilling hours, such as those located on the southern coast of Ecuador. Among all treatments evaluated the effects include direct application to the buds of hydrogen peroxide 5\%. This treatment gets stimulate bud breaking of almost all of the buds, advancing and increasing vegetative growth and doubling the performance of the strains, without compromising the quality of the grapes produced. Therefore we can say that the hydrogen peroxide treatments are a viable, economical and sustainable, alternative to $\mathrm{CH}$ applications in these culture conditions.
\end{abstract}

Keywords: Plant growth regulator, hydrogen peroxide, bud, toxicity, yield 


\section{Introducción}

El área tropical (entre 0-10 o latitud) está caracterizada por la ausencia de inviernos y temperaturas medias superiores a $20^{\circ} \mathrm{C}$ durante todo el año. Estas condiciones no satisfacen las necesidades de termorregulación y fotoperiodo de la vid. Esto induce desarreglos fisiológicos en la planta, que no pasa por periodos de reposo vegetativo, condicionando su manejo y limitando la vida útil del viñedo [1].

Las yemas requieren de la acumulación de un determinado número de horas frío para salir del periodo de endodormancia, Dokoozlian [2] menciona una demanda de 50 a 400 horas de frío dependiendo de la variedad de vid. La falta de acumulación de horas debajo de $7^{\circ} \mathrm{C}$ produce brotación desuniforme y retraso en la maduración de frutos [3], resultando necesario en zonas tropicales compensar esta ausencia total de horas de frío.

La utilización de $\mathrm{CH}$, con el objetivo de maximizar la brotación de las yemas y la sincronización de la floración, es una práctica extendida en la viticultura en condiciones tropicales, como las existentes en el litoral ecuatoriano. Aplicada directamente sobre las yemas se absorbe rápidamente, y cuando se metaboliza desencadena estrés oxidativo que conduce a la yema a salir de la endodormancia [4]. La CH causa la disminución de la actividad de la catalasa sin modificar la peroxidasa, lo que resulta en aumento de las concentraciones de peróxido de hidrógeno estimulando así la brotación [5]. Aunque tiene una alta eficacia, la $\mathrm{CH}$ es un producto altamente tóxico y está clasificado por la Agencia de Protección del Medio Ambiente de los Estados Unidos en la más alta categoría de toxicidad, Categoría I, además el registro de este producto es objeto de evaluación por parte de la Unión Europea [6]. Por lo tanto la exploración de tratamientos que substituyan a la $\mathrm{CH}$, que sean efectivos para inducir la ruptura de la dormición de las yemas, es imprescindible para el desarrollo de la viticultura en estas latitudes.

Se han estudiado diferentes alternativas a la $\mathrm{CH}$, aunque hasta el momento ninguno ha conseguido para estas condiciones un nivel de eficacia suficiente. Recientes investigaciones de Severo et al. [7] con aplicaciones de peróxido de hidrógeno $\left(\mathrm{H}_{2} \mathrm{O}_{2}\right)$ directamente sobre las yemas están dando resultados positivos en viñedos subtropicales de Brasil. Otros productos alternativos de bajo impacto como suplementos nutricionales están siendo desarrollados como Wert y Stimulant K, que han presentado buenos resultados para suplir la falta de horas de fríos en frutales como el cerezo. También Halaly et al. [8] han demostrado, en ensayos de invernadero, choques térmico puntual llegan a estimular la brotación de las yemas, manteniendo la yema a temperaturas de entre $50^{\circ} \mathrm{C}$ y $150^{\circ} \mathrm{C}$ variando el tiempo de exposición desde una hora hasta únicamente diez segundos, sin dañar el tejido y sin comprometer su viabilidad. Es imprescindible ajustar el protocolo de los tratamientos a condiciones de campo abierto y evaluar su eficacia en las diferentes ambientes de cultivo ya que existe una gran variabilidad según las condiciones concretas del viñedo.

El objetivo del proyecto fue evaluar la eficacia de diferentes tratamientos para estimular la brotación de las yemas y suplir la falta de horas de frío. Para ello se estudió el desarrollo y fenología de las yemas, el crecimiento vegetativo de los brotes, y finalmente el rendimiento y la composición fisicoquímica de la producción.

\section{Material y métodos}

Para alcanzar los objetivos marcados en el estudio se planteó un ensayo en un viñedo comercial de cv. Cabernet Sauvignon, injertada sobre IAC Jales 572. La parcela de ensayo, propiedad de Vitivinicola Del Morro, se estableció en el año 2009 ubicada en la parroquia del Morro, canton Guayaquil, provincia de Guayas (Ecuador). Las plantas están conducidas en doble Cordon Royat con una densidad de 2220 plantas/ha, habiendo una distancia de $1,5 \mathrm{~m}$. entre plantas y $3 \mathrm{~m}$. entre líneos. Se aplicaron cinco tratamientos además de un tratamiento testigo: Cianamida de hidrógeno (Dormex, Bayer) 5\%, Peróxido de hidrógeno 5\%; fitofortificante $10 \%$ (Wert, Tavan); Suplementos nutricionales 5\% (Stimulant K, Tavan) y tratamientos térmicos con agua a $80^{\circ} \mathrm{C}$. Todos ellos se evaluaron frente al tratamiento testigo de plantas sin tratar.

El diseño del ensayo se estableció en bloques completos al azar con cinco repeticiones, siendo la cepa la unidad experimental, en una zona de condiciones homogéneas y en cepas sanas de vigor equivalente. 
La aplicación de los productos fue realizado el $10 / 01 / 2015$, al día siguiente de haberse realizado la labores de poda, mediante pulverizadores manuales, realizando aplicaciones dirigidas exclusivamente a las yemas, con una gasto de 0,251 de volumen de caldo por cepa. Las temperaturas medias registradas durante el ciclo fueron de $26,4^{\circ}$, con máximas de $32,6^{\circ}$ y mínimas de $22,1^{\circ}$. La precipitación acumulada durante el ciclo fue de $159 \mathrm{~mm}$., concentrándose las tres cuartas partes del total durante el mes de marzo. Las plantas no se vieron expuestas a estrés hídrico ya que los abundantes aportes de riego por goteo fueron suficientes para cubrir las necesidades de agua.

A partir del tratamiento se realizaron semanalmente controles del estado fenológico, utilizando la escala BBCH [9] y también de la longitud del brote en cada una de las yemas de las cepas incluidas en el ensayo. En el momento de la vendimia se efectuaron controles del rendimiento y de sus diferentes componentes: número de racimos y de bayas y su peso correspondiente. Además se recogieron los racimos de cada tratamiento para recopilar una muestra de 500 bayas para extraer su mosto. Posteriormente tras el licuado, filtrado y centrifugado de las muestras, utilizando la centrifuga (Powerspin vx, Unico) durante $5 \mathrm{~min}$ a $3500 \mathrm{rpm}$, se analizó en el mosto su contenido en azúcar, mediante un refractómetro (HI-96801, Hanna instrument). También se determinó el pH y la acidez total, utilizando un pH-metro (BT-500, Boeco germany). Por último se determinó su intensidad y tonalidad colorante, mediante el método de Glories [10], por espectrofotómetría (AquaMate ${ }^{\mathrm{TM}}$ 2700E, Thermo Scientific). Con los datos recopilados en campo y en laboratorio se ejecutó el correspondiente análisis estadístico utilizando el paquete estadístico IBM SPSS Statistics 22.0, empleando los test de rango múltiple de Duncan $(\mathrm{p} \leq 0,05)$ para la comparación de las medias de los tratamientos.

\section{Resultados y discusión}

En cuanto a la fenología general del cultivo la duración media del ciclo fue de 135 días, alcanzando plena floración el 27/02/2015, a los 48 días después de la poda, y cosechándose el 25/05/2015. La brotación (ver Figura 1) de los plantas testigo y la de las plantas tratadas con productos fitofortificantes y suplementos nutricionales fue muy limitada, alcanzando valores cercanos al treinta por ciento. En el caso de las cepas a las que se realizó "choque térmicos" se produce un incremento significativo frente al testigo llegando a brotar más de la mitad de las yemas, en concordancia con lo observado por Halaly et al. [8]. Sin embargo, con el tratamiento con $\mathrm{CH}$ y también en el caso de aplicaciones de $\mathrm{H}_{2} \mathrm{O}_{2}$ estos porcentajes aumentaron significativamente hasta llegar a porcentajes de desborre de yemas cercanos al pleno. Esto reafirma la hipótesis planteada por Pérez et al. [5] según la cual aplicaciones exógenas de $\mathrm{H}_{2} \mathrm{O}_{2}$ en la endodormancia reproducen al menos parcialmente el efecto de la $\mathrm{HC}$.

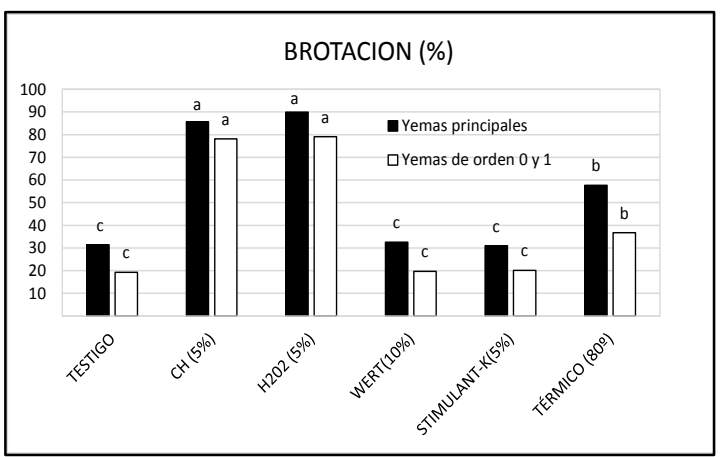

Figura 1. Porcentajes de brotación obtenidos en los distintos tratamientos reguladores de la brotación. Los valores con letra distinta son significativamente diferentes $(p<0,05)$

También Severo et al. [7] consiguieron resultados similares en esta misma línea, pero con concentraciones más elevadas de $\mathrm{H}_{2} \mathrm{O}_{2}$ $(10 \%)$ y en viñedos brasileños con temperaturas más moderadas. En cambio Potjanapimon et al. [11] en ensayos en condiciones controladas observó que la brotación en estaquillas tratadas con peróxido de hidrógeno $10 \%$ se inhibió ligeramente en comparación con las de control.

Por otra parte, cuando examinamos cuales de esas yemas brotadas están en una posición adecuada para poder renovar, tras la poda, a las yemas de este ciclo observamos que el porcentaje de las yemas tratadas con $\mathrm{CH}$ o con $\mathrm{H}_{2} \mathrm{O}_{2}$ llega hasta el ochenta por ciento del total, mientras que en el caso de los tratamientos testigos y los que tuvieron aplicaciones de Stimulant-K o Wert llegan únicamente al $20 \%$. Porcentajes cercanos al $40 \%$ tuvieron las yemas que fueron sometidas al choque térmico. La brotación de las yemas de madera vieja también se vio modificada significativamente por los tratamientos realizados. Mientras que las yemas sobre las que se había aplicado $\mathrm{CH}$ o peróxido de hidrógeno acumulaban 25 y 22 brotaciones de yemas, y las plantas expuestas a choques térmicos desarrollaban 17, los demás tratamientos se quedaban en una media de 12 , detectándose diferencias estadísticamente significativas $(\mathrm{p} \leq 0,05)$ entre los tres grupos. Este 
factor es muy importante para la viabilidad futura del cultivo ya que este tipo de brotaciones de madera vieja son utilizadas en muchas ocasiones para sustituir puestos de poda, evitando la degeneración del sistema de conducción del cultivo.

En cuando al desarrollo fenológico de esas yemas brotadas se observó un retraso significativo de entre tres y cinco días a partir de la emergencia de las hojas (estado 11) [9] de las plantas tratadas con $\mathrm{CH}$ con $\mathrm{H}_{2} \mathrm{O}$, con respecto a los controles y al resto de tratamientos evaluados. Este retraso condicionó el crecimiento vegetativo de los sarmientos, por lo cual alcanzaron longitudes finales del sarmiento significativamente mayores en el caso de tratar con $\mathrm{CH}(99 \mathrm{~cm})$, y también con $\mathrm{H}_{2} \mathrm{O}_{2}(97 \mathrm{~cm})$ y con choques térmicos $(91 \mathrm{~cm})$ frente a los testigos que se quedaron limitados a $71 \mathrm{~cm}$. Los demás tratamientos tuvieron un crecimiento vegetativo similar al de las plantas sin tratar.

La producción de las plantas (ver Figura 2) se vio significativamente alterada por la distintas aplicaciones realizadas. Se distinguieron dos grupos bien diferenciados, los plantas testigo y las tratados con Wert, Stimulant-K o sometidas a tratamiento térmico obtuvieron rendimientos más reducidos, mientras que las plantas tratadas con $\mathrm{CH}$ y con $\mathrm{H}_{2} \mathrm{O}_{2}$ doblaron su producción frente a las del grupo anterior, consiguiendo rendimientos de casi 4kg/cepa. Esta mayor productividad observada en las plantas tratadas con $\mathrm{CH}$ y $\mathrm{H}_{2} \mathrm{O}_{2}$, fue consecuencia de la mayor la superficie foliar expuesta [12].

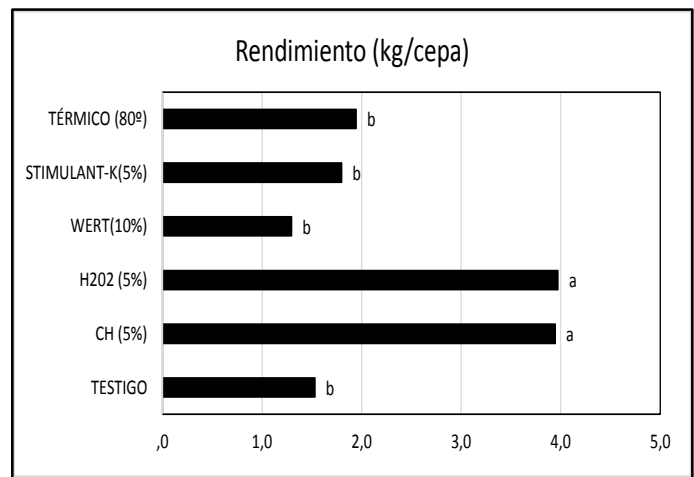

Figura 2. Valores de rendimiento obtenidos en los distintos tratamientos reguladores de la brotación. Los valores con letra distinta son significativamente diferentes $(p<0,05)$.

El incremento en el rendimiento fue debido al aumento significativo del número de racimos por planta (ver Figura 3) ya que el resto componentes del rendimiento, tanto el número como el peso de las bayas, no varió significativamente con la aplicación del tratamiento.

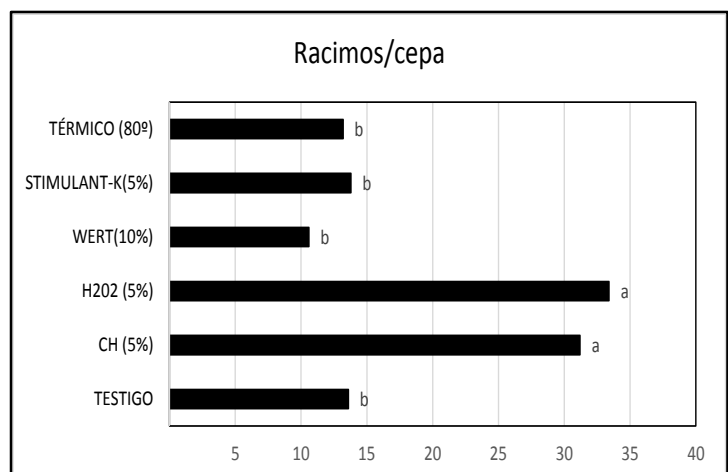

Figura 3. Valores de rendimiento obtenidos en los distintos tratamientos reguladores de la brotación. Los valores con letra distinta son significativamente diferentes $(p<0,05)$

Respecto a la composición físico-química de la bayas (ver Tabla 1) no se presentaron diferencias estadísticas significativas.

Tabla 1. Valores medios de la composición fisicoquímica de las bayas en los distintos tratamientos reguladores de la brotación de las yemas.

\begin{tabular}{lccccc}
\hline Tratamientos & ${ }^{\circ}$ Brix & $\mathrm{pH}$ & $\begin{array}{c}\text { Acidez total } \\
\text { (g tartárico/l) }\end{array}$ & $\begin{array}{c}\text { Intensidad } \\
\text { colorante }\end{array}$ & $\begin{array}{c}\text { Tonalidad } \\
\text { colorante }\end{array}$ \\
\hline \hline Testigo & 14,69 & 3,71 & 3,11 & 4,53 & 1,45 \\
$\mathrm{CH}(5 \%)$ & 13,82 & 3,78 & 2,88 & 4,94 & 1,68 \\
$\mathrm{H}_{2} 0_{2}(5 \%)$ & 16,39 & 3,85 & 2,73 & 5,42 & 1,50 \\
$\begin{array}{l}\text { Wert(10\%) } \\
\text { Stimumant- }\end{array}$ & 14,18 & 3,79 & 2,70 & 5,63 & 1,43 \\
$\begin{array}{l}\mathrm{K}(5 \%) \\
\text { Térmico } \\
\left(80^{\circ}\right)\end{array}$ & 15,45 & 3,76 & 3,13 & 5,76 & 1,35 \\
\hline
\end{tabular}

Este fenómeno se explica porque el menor rendimiento de las plantas compensó la reducción del desarrollo vegetativo y por tanto de un potencial fotosintético menor, provocando que la planta sea capaz de presentar niveles de maduración similares. Igualmente la producción obtenida consigue las características fisicoquímicas exigidas por el mercado.

\section{Conclusiones}

A la vista de los resultados obtenidos en el ensayo podemos afirmar que en nuestras condiciones de estudio, los tratamientos de con peróxido de hidrógeno al $5 \%$ son capaces de estimular la brotación de las yemas con un nivel de eficacia similar que el tratamiento usualmente utilizado con $\mathrm{CH}$ al $5 \%$. Asimismo asegura el mantenimiento del sistema de conducción en los siguientes ciclos de cultivo, ya que presenta una brotación suficiente de yemas 
adecuadas para la renovación. Al mismo tiempo las plantas a las que se aplicó este tipo de tratamientos ven doblada su producción y por tanto su rentabilidad, sin ver comprometida con ello la calidad de la cosecha. Los choques térmicos sobre las yemas tienen un efecto estimulador de la brotación, pero este no es tan efectivo y regular como en caso de la $\mathrm{CH}$, y tampoco logra incrementar el rendimiento de las plantas.

Aunque hay que continuar con las investigaciones en esta línea para que los resultados sean concluyentes, los obtenidos en este estudio invitan a creer que la $\mathrm{H}_{2} \mathrm{O}_{2}$ puede ser un substituto real de la $\mathrm{CH}$. Sería de gran interés hacer ensayos en otras parcelas para confirmar los resultados y realizar ensayos de dosis para ver si es posible reducir la

\section{Referencias}

[1] Fregoni, M. 2005. La geografía mondiale delle uve da tavola. L'Informatore Agrario 48: Supplemento n.1 Uva da tavola, 11.

[2] Dokoozlian, N.K. 1999. Chilling temperature and duration interact on the budbreak of 'Perlette' grapevine cuttings. HortScience 34(6), 1054-1056.

[3] Almazan, P.J., Quijano, M.A. Fisher, G., Chaves, B. y Balaguera, H.E. 2010. Physicochemical characterization of Pinot Noir grapevine (Vitis vinifera L.) fruit during its growth and development under high altitude tropical conditions. Agron. Colomb. 28(2), 173-180.

[4] Ben Mohamed, H.; Zrig, A.; Geuns, J. M. C.; Khemira, H. 2014. Near-lethal heat treatment induced metabolic changes associated with endodormancy release of Superior Seedless grapevine cv. (*Vitis vinifera* L.) buds. Australian Journal of Crop Science. Source: 8 (4) 486-494.

[5] Settimi, L.; F. Davanzo; M. Faraoni; G. Micelli; D. Richmond y G. Calvert. 2005. Hidrogen cyanamide-related illnesses-Italy, 2002-2004. Morbidity and Mortality Weekly Report, Atlanta 54, 405-408 concentración de las aplicaciones sin ver disminuida su eficacia. Este cambio en la materia activa empleada evitaría los riesgos de intoxicación a los que se ven expuestos los operarios que manipulan $\mathrm{CH}, \mathrm{y}$ muy probablemente los costos de la aplicación se verían reducidos sensiblemente

\section{Agradecimientos}

Este trabajo se ha realizado en el marco del Proyecto Prometeo, financiado por la Secretaria nacional de eduación superior ciencia tecnología e innovación (SENESCYT) de Ecuador, dentro del proyecto "Mejora de la calidad de la uva de vinificación procedente de viñedos ecuatorianos mediante la utilización de reguladores del crecimiento y nuevas técnicas de cultivo".

[6] Severo F., Severo R., Rangel L., Ferreira S.M., Pereira A., Saavedra J. 2014. Breaking the dormancy bud on rootstock 'SO4'. Proceeding $34^{\text {th }}$ Congress OIV Mendoza (Argentina)

[7] Halaly, T.; Zion, B.; Arbel, A.; Regev, R.; Barak, M.; Or, E. 2011. Short exposure to sublethal heat shock facilitates dormancy release in grapevines. American Journal of Enology and Viticulture. Source: 62 (1) 106-112.

[8] Pérez, F., Vergara, R. y Rubio, S. 2008. $\mathrm{H}_{2} \mathrm{O}_{2}$ is involved in the dormancy-breaking effect of hydrogen cyanamide in grapevine buds. Plant Growth Regulator. 55:149-155.

[9]Lorenz DH, Eichhorn KW, Bleiholder H, Klose R, Meier U, Weber E. 1995. Growth stages of the grapevine: Phenological growth stages of the grapevine (vitis vinifera 1 . ssp. vinifera)-codes and descriptions according to the extended bbch scale. Aust J Grape Wine Res. 1(2):100-3.

[10] Glories, Y. 2001. Caractérisation du potentiel phénolique: adaptation de la vinification. Progrès Agricole et Viticole, $118,15 / 16347-350$. 
[11] Potjanapimon, C.; Fukuda, F.; Kubota, N. 2007. Effects of various chemicals and their concentrations on breaking bud dormancy in grapevines. Scientific Reports of the Faculty of Agriculture, Okayama University. 96: 19-24.
[12] González, R. 2012. Utilización de etefón, etanol y ácido abscísico para controlar el desarrollo del viñedo y mejorar la calidad de la uva cv. Verdejo. Tesis doctoral. Dpto. Producción Vegetal. Universidad de Valladolid. 